\title{
Development of Nursing Protocol for Preventing Discontinuation of Treatments by Methods Other than Physical Restraint during Acute Exacerbation of Chronic Heart Failure in Patients with Impaired Cognitive Function
}

\author{
Haruka Otsu ${ }^{*}$, Shiori Fujimoto², Nozomi Murakami ${ }^{3}$, Tatsuki Ohhara4, Yoko Takeya5, \\ Tatsuya Ohno', Chieko Suzuki ${ }^{7}$, Sanae Takahashi8 \\ ${ }^{1}$ Graduate School of Health Sciences, Hirosaki University, Hirosaki City, Japan \\ ${ }^{2}$ Japan Community Health Care Organization, Shiga Hospital, Shiga, Japan \\ ${ }^{3}$ Akita Prefecture Health Agricultural Cooperative Association, Hiraka General Hospital, Yokote, Japan \\ ${ }^{4}$ Akita Prefecture Health Agricultural Cooperative Association, Koto Kosei Hospital, Hachirogata, Japan \\ ${ }^{5}$ Aomori Prefectural Central Hospital, Aomori, Japan \\ ${ }^{6}$ Medical Corporation Association Seikokai, Hirano Synthesis Hospital, Gifu, Japan \\ ${ }^{7}$ Ugo Municipal Hospital, Ugo, Japan \\ ${ }^{8}$ Omagari Nakadori Hospital, Omagari, Japan \\ Email: `h_otsu@hirosaki-u.ac.jp, oryokuchan@icloud.com, nzmooi1020mrkmuaai@lapis.plala.or.jp, nurse.mgr@koto-ghp.jp, \\ x5452ta@ybb.ne.jp,nsoono1022@gmail.com, inzatat@yutopia.or.jp,o-hns3@meiwakai.or.jp
}

How to cite this paper: Otsu, H., Fujimoto, S., Murakami, N., Ohhara, T., Takeya, Y., Ohno, T., Suzuki, C. and Takahashi S. (2018) Development of Nursing Protocol for Preventing Discontinuation of Treatments by Methods Other than Physical Restraint during Acute Exacerbation of Chronic Heart Failure in Patients with Impaired Cognitive Function. Health, 10, 789-815.

https://doi.org/10.4236/health.2018.106060

Received: June 4, 2018

Accepted: June 22, 2018

Published: June 25, 2018

\begin{abstract}
The purpose of this study is to prepare a nursing protocol for preventing discontinuation of treatments using a method other than physical restraint during acute exacerbation of chronic heart failure in patients with impaired cognitive function. For the first stage of the study, we prepared a draft of the nursing protocol based on a basic survey. For the second stage, semi-structured interviews were conducted with 5 nurses specialized in chronic heart failure and 10 nurses in dementia case to ensure content validity of the draft protocol. For the third stage, we examined the possibility of clinical application of the revised version of the protocol draft prepared in the second stage of the study. For assessment items, significant points of nursing care, and specific nursing care practice in this revised version, 154 subjects (93.9\%) considered effective for patients, in terms of prevention of treatment discontinuation using a method other than physical restraint. All items and contents were considered useful by more than $60 \%$ of the nurses. Considering that the nurses working in the clinical setting reported $93.9 \%$ of usefulness, we concluded that this nursing protocol remained valid at a certain level. We also received a
\end{abstract}


Copyright (c) 2018 by authors and Scientific Research Publishing Inc. This work is licensed under the Creative Commons Attribution International License (CC BY 4.0).

http://creativecommons.org/licenses/by/4.0/

Open Access comment from the certified nurses that we should include the basic contents for newly graduated nurses. We consider that this nursing protocol will be also useful for newly graduated nurses to acquire knowledge. It helped to standardize nursing care in order to predict potential risks for patients with impaired cognitive function.

\section{Keywords}

Dementia, Impaired Cognitive Function, Acute Exacerbation of Chronic Heart Failure, Physical Restraint, Nursing Protocol

\section{Introduction}

About $80 \%$ of elderly patients with dementia who are in the acute exacerbation phase of heart failure are likely to develop behavioral psychological symptoms of dementia (BPSD) such as restlessness, agitation, and hyperactivity as they hardly understand the necessity of disease management and feel stressed against invasive treatments [1]-[6]. As elderly patients with dementia with heart failure in the acute exacerbation phase are at high risk of treatment discontinuation, nurses face a difficulty dealing with these patients to continue treatments of heart failure [1]-[6]. Although some attempts have been made in order to prevent discontinuation of treatments, physical restraint has been still used in some medical institutions to continue the treatments [1]. Physical restraint makes patients restless or agitated, imposes an excessive cardiac load causing deterioration of heart failure, and increases the risk of physical injury due to falls; these have been the adverse effects of this method [1]. In the year of 2016, a revision of the medical payment system was made in Japan, in a way that the number of points added is reduced by $40 \%$ per day if they performed physical restraint to the elderly patients with dementia who have symptoms categorized to the ADL criteria level III (which interfere with activities of daily living, showing difficulty in activities and communications and requiring nursing care) or above [7]. Therefore, it is required for medical institutions to develop nursing care other than physical restraint with which they can continue treatments for acute exacerbation of physical disorders in elderly people with dementia.

For the methods to prevent discontinuation of treatments for acute exacerbation of heart failure in the elderly patients with dementia, Otsu et al. conducted a survey on nurses working at hospitals with cardiovascular ward with experiences of cardiac failure treatments and on nurses specialized in dementia care with experiences in nursing care of demented elderly patients with cardiac failure; In this study, they investigated situations that the nurses felt difficult to deal with and the methods that they thought effective for such situations [1] [8]. However, the subjects included only a few nurses specialized in chronic heart failure, who have expert knowledge in nursing care for heart failure. In addition, there was a limit in this study to actually develop the effective method as it was a 
self-administrated questionnaire survey. The focus of this study was on nursing care for patients who have symptoms of both chronic heart failure and dementia; hence, it is essential to capture characteristics of nursing care for both diseases and their symptoms. With this background, we considered that we need to develop a nursing protocol from perspectives of nurses specialized in chronic heart failure and dementia care, who are the specialists for nursing care in chronic heart failure and dementia, in order to prevent discontinuation of treatments for acute exacerbation phase of heart failure in patients with impaired cognitive function using a method other than physical strain. Standardization of nursing seems to be meaningful in preventing evils resulting from physical restraint, improving patient's QOL and reducing mental suffering in families. We believe that this protocol will be meaningful to prevent adverse effects of physical restraint, improve the patients' QOL and reduce psychological distress of the families. The purpose of this study is to develop a nursing protocol to prevent discontinuation of treatments with methods other than physical restraint during acute exacerbation of chronic heart failure in patients with impaired cognitive function.

\section{Methods}

For the first step of the research, a draft of the nursing protocol was prepared based on a basic survey [4] for patients with chronic heart failure with impaired cognitive function. For the second stage, semi-structured interviews were conducted with 5 nurses specialized in chronic heart failure and 10 nurses in dementia care to ensure the content validity of the draft protocol. We asked candidates to participate in a certified nurses' education curriculum and we were introduced to certified nurses in both fields. In the interviews, we asked their opinions on characteristics of patients with impaired cognitive function with acute exacerbation of chronic heart failure [4] as well as on the draft of the protocol. Then we revised the draft according to their opinions. Each interview lasted for about 120 minutes and was conducted in a private room.

For the third stage, we examined the possibility of clinical application of the revised draft proposed in the second stage. We confirmed usefulness of the revised draft through actual cases that they experienced and completed the nursing protocol. The subjects had been working at 70 hospitals with cardiovascular ward, where they had a delirium prevention team and nurses specialized either in chronic heart failure or dementia. They all had experiences of nursing care in patients with impaired cognitive function with acute exacerbation of chronic heart failure. We requested nursing administrators of these hospitals in a written form to cooperate with us in this study, and for those who agreed, we asked them to send us replies regarding the number of copies of the survey forms that they need to distribute. The investigation was conducted during October and November 2017. The subject were asked to select all the items that they felt useful when assessing interruptions during the treatments as well as the care that 
they provided in order to successfully prevent the interruptions during the treatment.

\section{Analysis Method}

In the second stage of the study, we conducted an analysis using qualitative induction on data obtained by the interviews. We created verbatim records and extracted descriptions concerning the evaluation of the draft protocol. The extracted contents were coded and classified into similar semantic contents.

In the third stage, we performed a simple tabulation and counted the numbers of assessment items and nursing care contents selected by the nurses as useful. The items and contents selected by more than $60 \%$ of nurses were considered valid to be included in the nursing protocol. For items selected by less than $60 \%$ of nurses, we judged if the patients' heart failure could be aggravated by implementation of the nursing protocol. If the possibility was low, it was assumed that the protocol was not properly implemented as the subjects did not have adequate expert knowledge or skills in nursing care of chronic heart failure and dementia. Hence, we included these items in the nursing protocol as assessment items and nursing care contents.

\section{Ethical Consideration}

In the second and third stages, we explained the purpose, method, research contents, voluntariness of participation in the study, and protection of personal information to the nurses and nursing administrators in a written form. We conducted the investigation under the subjects' voluntary agreement. This research was approved by the Ethics Committee of the Graduate School of Health Sciences, Hirosaki University (Reference Number: 2015-047).

\section{Results}

\subsection{Results of the First Stage}

A draft of the nursing protocol was prepared based on the basic survey [4] and the guidelines for treatments of acute heart failure [5] in order to prepare a nursing protocol for treatments frequently performed for acute exacerbation phase of chronic heart failure in patients with impaired cognitive function. In the basic survey [4], we revealed issues in nursing care which make it difficult for patients with impaired cognitive function to continue treatments for acute exacerbation of chronic heart failure or to go through monitoring of symptoms. These issues included the following: 1) inadequate adaptation to the environment due to sudden hospitalization, 2) anxiety due to insufficient understanding regarding clinical examinations, management and treatments, 3) delay in recovery due to interruption of treatments and monitoring in the acute phase, 4) difficulty in capturing symptoms associated with deterioration of heart failure, 5) difficulty in monitoring symptoms when using diuretics, 6) difficulty in adhering to diet and fluid-intake restriction. Assuming that these problems would oc- 
cur in time series through the process of hospitalization, clinical examination, treatment, and symptom monitoring, we created a chronological flowchart. On the left side of the flow chart, we assumed the problems in nursing care which could occur at the beginning of hospitalization and conducted an assessment following the assessment items. In case where there was a possibility of having problems or risks, we created a summary of specific nursing care to be conducted following the significant points of nursing care on the right side of the chart. Regardless of the number of years of nursing experience, we listed specific nursing care contents to be conducted so that they could at least provide minimum nursing care.

\subsection{Results of the Second Stage}

\subsubsection{Outline of Target}

Table 1 shows the outline of the certified nurses who were the subjects of this study. A total of 15 people, including 5 certified chronic heart failure nurses and 10 nurses with dementia nursing care experience, were eligible. Age was $43.2 \pm$ 5.7 years for chronic heart failure nurses and $39.5 \pm 10.0$ years for certified dementia nurses. Average years of experience as a nurse was $19.0 \pm 7.5$ for chronic heart failure nurses and $18.3 \pm 6.1$ years for certified dementia nurses. The years of experience of certified nurses were $3.4 \pm 0.5$ years for certified chronic heart failure nurses and $5.0 \pm 1.6$ years for certified dementia nurses. Five in certified chronic heart failure nurses was females and for certified dementia nurses, it was 8 females and 2 males.

\subsubsection{Evaluation of the Draft of the Nursing Protocol by Nurses Specialized in Chronic Heart Failure and/or Dementia Care}

The opinions of nurses specialized in chronic heart failure regarding the entire protocol draft are shown in Table 2. It included 27 codes and 17 categories.

The opinions of nurses specialized in dementia care regarding the entire protocol draft are shown in Table 3. It included 94 codes and 38 categories.

\subsubsection{The Possibility of Delayed Recovery Due to Discontinuation of Treatments and Symptom Monitoring in the Acute Phase}

The opinions of nurses specialized in chronic heart failure and/or dementia care regarding the possibility of delayed recovery due to discontinuation of treatments and symptom monitoring in acute phase are shown in Table 4.

\subsubsection{Difficulty in Monitoring Patients When Using Diuretics}

The opinions of nurses specialized in chronic heart failure and/or dementia care regarding the difficulty in monitoring patients when using diuretics are shown in Table 5.

\subsection{Results of the Third Stage}

\subsubsection{The Number of the Survey form Distributed and Collected}

The form was sent to nursing administrators of 70 institutions. Of these, 31 institutions responded to help us in this study. We asked the nursing administrators 
Table 1. Outline of target.

\begin{tabular}{cccccc}
\hline Nurse & Sex & Type of certified nurse & Age & $\begin{array}{c}\text { No. of years of experience } \\
\text { (years) }\end{array}$ & $\begin{array}{c}\text { No. of years of experience as a } \\
\text { certified nurse (years) }\end{array}$ \\
\hline A & Female & CHF nursing & 30 's & 13.3 & 3 \\
B & Female & CHF nursing & 40 's & 13 & 4 \\
C & Female & CHF nursing & 40 's & 18.5 & 3 \\
D & Female & CHF nursing & 40 's & 18.5 & 3 \\
E & Female & CHF nursing & 50 's & 31.5 & 4 \\
F & Female & Dementia nursing & 30 's & 13.4 & 8 \\
G & Female & Dementia nursing & 40 's & 21.5 & 5 \\
H & Female & Dementia nursing & 40 's & 21.5 & 5 \\
I & Female & Dementia nursing & 30 's & 13.3 & 4 \\
J & Male & Dementia nursing & 40 's & 24.7 & 4 \\
K & Female & Dementia nursing & 40 's & 20.8 & 4 \\
L & Female & Dementia nursing & 40 's & 19.6 & 4 \\
M & Male & Dementia nursing & 30 's & 10.6 & 4 \\
N & Female & Dementia nursing & 30 's & 16.7 & 8 \\
O & Female & Dementia nursing & 40 's & 26.9 & 4 \\
\hline
\end{tabular}

Table 2. The opinions of nurses specialized in chronic heart failure regarding the entire protocol draft.

\begin{tabular}{|c|c|}
\hline Categories & Number of codes \\
\hline The priority should be given in order of oxygen therapy, drug therapy, and resting therapy & 4 \\
\hline We should not use drugs without much consideration against BPSD that may cause disease aggravation & 3 \\
\hline $\begin{array}{l}\text { We should divide the nursing protocol into three phases: the early days of hospitalization, acute phase and recovery } \\
\text { phase. }\end{array}$ & 3 \\
\hline The nursing protocol will be useful for newly graduated nurses. & 2 \\
\hline We want to use the nursing protocol as soon as possible. It will be helpful. & 2 \\
\hline We will use the nursing protocol once the patients are transferred to the ward. & 1 \\
\hline We should have priority order for contents of the nursing protocol. & 1 \\
\hline We should include the use of diuretic in items for monitoring. & 1 \\
\hline We should not to limit the place where we use the nursing protocol. & 1 \\
\hline The contents should be simple. & 1 \\
\hline We should have the implementation criteria for physical restraint. & 1 \\
\hline $\begin{array}{l}\text { The subjects in the nursing protocol should include those who cannot continue a conversation or those with } \\
\text { disorientation. }\end{array}$ & 1 \\
\hline Diuretics are not used for all patients. & 1 \\
\hline $\begin{array}{l}\text { It may be safe to use sedatives and hypnotics which impose relatively less stress on the heart for about } 3 \text { days after } \\
\text { hospitalization for patients' restlessness or excitement. }\end{array}$ & 1 \\
\hline For patients with agitation or insomnia, we should give medication after dinner or at bedtime. & 1 \\
\hline It is difficult to increase diuretics if patients cannot tolerate fluid restriction. & 1 \\
\hline $\begin{array}{l}\text { I am not sure if we should apply a cold pad to the perineal area to reduce the discomfort of the bladder indwelling } \\
\text { catheter. }\end{array}$ & 1 \\
\hline The nursing protocol contains too much contents. & 1 \\
\hline
\end{tabular}


Table 3. The opinions of nurses specialized in dementia care regarding the entire protocol draft.

\begin{tabular}{|c|c|}
\hline Categories & Number of codes \\
\hline The priority should be given in order of oxygen therapy, drug therapy, and resting therapy & 14 \\
\hline We should divide the nursing protocol into three phases: the early days of hospitalization, acute phase and recovery phase. & 9 \\
\hline It will be easier to implement the nursing protocol in the clinical setting if it is described in detail. & 6 \\
\hline We should divide the nursing protocol into two parts: significant points and implementation procedure in detail. & 6 \\
\hline The simpler it is, the easier to read and follow. & 5 \\
\hline Contents of the nursing protocol are much better. & 4 \\
\hline The order and flow of the nursing protocol seems reasonable. & 4 \\
\hline The contents of the nursing protocol should be expressed in such an applicable way that it can be applied. & 4 \\
\hline We need descriptions regarding how to cope with memory impairment in the nursing protocol. & 3 \\
\hline We should have priority order for contents of the nursing protocol. & 3 \\
\hline The nursing protocol will be useful. & 2 \\
\hline Urinary tract infection frequently occurs in patients during hospitalization. & 1 \\
\hline We should have a summary of the nursing protocol to show significant points. & 1 \\
\hline It is good to make assessments based on yes or no and proceed to nursing care. & 1 \\
\hline We should include dehydration index in the nursing protocol. & 1 \\
\hline Abstract contents may be easier to apply to the practice. & 1 \\
\hline We should not specify the place where we use the nursing protocol. & 1 \\
\hline We will need to consider the timing of releasing physical restrain. & 1 \\
\hline We should include drug adjustment for patients with restlessness and agitation. & 1 \\
\hline I would like to try to apply a hot towel around the neck of the patient. & 1 \\
\hline We should include the basic contents as well for newly-graduated nurses. & 1 \\
\hline $\begin{array}{l}\text { We should create a nursing protocol along with the time course of symptoms observed and treatments provided after } \\
\text { hospitalization. }\end{array}$ & 1 \\
\hline The nursing protocol appears practical & 1 \\
\hline
\end{tabular}

Table 4. The opinions of nurses specialized in chronic heart failure and/or dementia care regarding the possibility of delayed recovery due to discontinuation of treatments and symptom monitoring in acute phase.

Nurses specialized in chronic heart failure
We should include "maintenance of bed-resting and prevention of interruption" in the specific nursing aids.
We must consider what to focus on not only for dementia but also for the level of cardiac functions.
We should include Metabolic equivalents (METs) as an indicator of heart failure nursing protocols for activity.
We should include in the nursing protocol appropriate activity level with METs for demented patients.
Activity assessment varies among nurses; We should include activities in the nursing protocol.
Therapeutic meal is provided from the first day of hospitalization; If the patient does not eat, enteral feeding is performed. If the patient is under
BiPAP (Bilevel positive airway pressure) therapy, the tube is removed during a meal.

to distribute a total of 272 copies of the survey form. A total of 164 copies, with the recovery rate of $60.3 \%$ were collected from the subjects. The number of valid responses was 164 , and the effective response rate was $60.3 \%$. 
Table 5. The opinions of nurses specialized in chronic heart failure and/or dementia care regarding the difficulty in monitoring patients when using diuretics.

Nurses specialized in chronic heart failure

Diuretic is indispensable for treatment of acute chronic heart failure, so it must be included in the nursing protocol. Insertion of a bladder indwelling catheter is necessary not only for monitoring symptoms but also for bed-resting therapy.

After starting diuretic therapy, we need to pay attention to the patient's excretion behavior especially within 30 minutes with IV.

Whether or not the patient needs the bladder indwelling catheter insertion and its duration vary according to the bed-resting level in the cardiac function evaluation.

I have never cooled down the patient's perineal area. The effect is unknown.

If the cardiac load does not become excessive within the physical activity level 2 - 3 with METs, we should remove the bladder indwelling catheter. We should speak with the physician and discuss how much the patient needs to rest or if he/she needs the bladder indwelling catheter.

There is always the possibility that the patient removes the bladder indwelling catheter by him/herself. Special care should be taken when a large amount of diluted urine flows out after intravenous diuretic administration. We can prevent the self-removal of the catheter by showing the patient anytime that the diluted urine has been flowing well, by milking the catheter if it's flowing.

We should try to cool down the perineal area to alleviate the discomfort.

Carperitide (Hanp) has been used for 24 hours during the first 3 - 4 days on average after hospital admission. We no longer use this for more than one week.

Nurses specialized in dementia care

If the patient frequently shows his/her desire to void, we should try to measure the urine volume in the bladder using a bladder scan and consider if urination induction is necessary.

\subsubsection{An Overview of the Participants}

As for the number of years of nursing experience, 6 nurses (3.7\%) had 1 year experience, 7 nurses (4.3\%) had 2 - 3 years, 20 nurses (12.2\%) had 4 - 5 years, 30 nurses (18.3\%) had 6 - 10 years, 60 nurses (36.6\%) had $11-20$ years, 31 nurses (18.8\%) had 21 - 30 years, and 10 nurses (6.1\%) had over 31 years of experience. The subjects included 141 females (86.0\% and 23 males (14.0\%).

\subsubsection{Evaluation of Usefulness of the Revised Draft of the Nursing Protocol}

According to the subjects, 3 assessment items and 5 specific nursing-care contents were not effective for patients (Table 6). These items and contents however were considered valid in the second stage of this study in cases experienced by the nurses specialized in chronic heart failure or dementia care. Therefore, we did not eliminate those items and contents. For all other items and contents, 154 (93.9\%) nurses considered effective for their patients.

Based on the results of the second and the third stages, we modified the revised version of the nursing protocol. Then, we created the nursing protocol for preventing discontinuation of treatments by methods other than physical restraint during acute exacerbation of chronic heart failure in patients with impaired cognitive function (Figure 1). This protocol is implemented when patients need to receive oxygen therapy or continue IV drip. After implementing this nursing protocol, it is necessary to evaluate whether the frequency of discontinuation of treatment decreases for patients with impaired cognitive function.

Specific nursing care contents in the preliminary version of the nursing protocol to prevent discontinuation of treatments by methods other than physical 
Table 6. Items and contents conducted by nurses but were not effective for patients.

\begin{tabular}{|c|}
\hline Assessment items \\
\hline 3. Interruption of bed-resting therapy associated with voiding behavior and its risk \\
\hline 1) High risk condition \\
\hline$\square \quad$ Have a strong sense of shame \\
\hline$\square \quad$ Dirty and filthy appearance with smell at hospital admission (high risk of urinary tract infection) \\
\hline 4) Some sort of needs \\
\hline$\square \quad$ Sexual deviation behavior \\
\hline Specific Nursing Care Practice \\
\hline $\begin{array}{l}\text { 3. The amount and frequency of urine excretion increase in patients with diuretics; once we remove the catheter, they will frequently repeat } \\
\text { voiding behavior/action. This may cause increase of cardiac stress, leading to aggravation of heart failure. }\end{array}$ \\
\hline 2) Reduction of unpleasant and discomfort feeling associated with the bladder indwelling catheter \\
\hline$\square$ Use bladder indwelling catheter made of silicone with a sufficient amount of lubricant at insertion. \\
\hline 3) Reinforcement of observation to prevent the patients' self-removal of the catheter. \\
\hline $\begin{array}{l}\text { If the patient frequently shows his/her desire to void, measure the urine volume in the bladder using a bladder scan and consider if } \\
\text { urination induction is necessary. }\end{array}$ \\
\hline 8) Attempt to prevent the patients' self-removal of the catheter so that they will not have excessive cardiac load. \\
\hline $\begin{array}{l}\text { Write down on a piece of paper at what time the patient voided last time, and let the patient know that he/she just finished voiding } \\
\text { by showing a clock and the paper. }\end{array}$ \\
\hline 4. The patients' cardiac load may increase when they move to the toilet, resulting in aggravation of heart failure. \\
\hline 1) Attempt to avoid excessive cardiac load \\
\hline If the patient has been extracting stool by him/herself, ask if a nurse can do it for him/her. \\
\hline 10. If the symptoms are overlooked, aggravation of heart failure may not be detected in the early stage. \\
\hline (1) Attempt to detect and manage aggravation signs in the early stage. \\
\hline$\square \quad$ Nurses who know the characteristics of the patient, such as primary nurses, should deal with the case. \\
\hline
\end{tabular}

restraint during acute exacerbation of chronic heart failure in patients with impaired cognitive function.

1) The patient is in a hypoxic state; there is a risk of delayed recovery of heart failure

[The significant point of nursing aid].

$\square$ Improving pulmonary congestion increases the awareness level and comprehension ability. Prevent the patient from worrying about oxygen therapy and prevent interruptions.

a) Reduce uncomfortable feeling of NPPV and the use of an oxygen mask

Use a mask with less discomfort such as an oxy mask for the patient.

$\square$ If the patient removes NPPV, use oxygen mask, Nasal High Flow, or Nasal oxygen cannula to continue oxygen therapy.

$\square$ If the patient feels discomfort to Nasal oxygen cannula, cut the tip of the cannula so that the cannula does not touch the patient's nasal cavity.

$\square$ Release the rubber for fixing the mask and cannula and relieve the pressure on the patient. 
All of the following high-risk conditions

$\square$ Moderate in dementia $\square$ Consciousness level tends to recover as soon as possible after hospitalization

1. Oxygen therapy interruption and its risk

1) Uncomfortable feeling of oxygen therapy

口Oppression and discomfort of invasive positive pressure ventilation (NPPV) or oxygen mask

口Touch NPPV or oxygen mask

$\square$ Remove NPPV or oxygen mask

2) Exacerbation of heart failure symptoms and distress

$\square$ Declining consciousness level due to hypoxic condition

$\square$ Restlessness and uneasiness due to breathing difficulty

$\square$ Pain that cannot be restored by taking the position of the lying seat

\section{No}

2. Interruption of drug therapy and its risk

1) High risk condition

$\square$ Signs and actions of oxygen therapy interruption

$\square$ Touch intravenous line and penetration part

$\square$ Use of drugs such as humps that may reduce blood pressure

$\square$ There is a possibility of vascular pain using antibiotics

$\square$ Thin blood vessels are used

$\square$ There is a possibility of drip leak

2) Interruption sign

$\square$ Attempt to remove self-infusion

No

3. Interruption of resting therapy related to voiding behavior and its risk

1) High risk condition

$\square$ signs of oxygen therapy interruption

$\square$ Rapid diuresis or diluted urine at the time of using diuretic drugs

$\square$ Prostatic Hypertrophy

$\square$ Overactive bladder

2) Personal background

$\square$ Alone $\quad \square$ Self-support is strong $\square$ Shame is strong $\square$ Male

$\square$ Individual excretion behavior before hospitalization is independent

$\square$ Tendency to hold back $\quad \square$ Desire to excrete in the toilet

$\square$ The appearance at the time of admission is bad and it smells (high risk of urinary tract infection)

3) Uncomfortable feeling

$\square$ Discomfort of bladder indwelling catheter

$\square$ Use of rubber catheter

$\square$ Indication of urinary urgency during insertion of the bladder indwelling catheter

4) Excretion needs

$\square$ Ask for assistance such as "Someone" "Help me" "Nurse"

$\square$ Landing behavior such as side sitting position, standing position, wandering

$\square$ Spontaneous movements to the toilet

$\square$ Appeal against bed excretion

$\square$ There is sexual deviation behavior such as touching genitals

4. Interruption of resting therapy related to defecation behavior and its risk

1) High risk condition

$\square$ The patient can not convince bed defecation

$\square$ constipation from before hospitalization

2) Personal background

$\square$ Alone $\square$ Self-support is strong $\square$ Shame is strong

$\square$ Individual excretion behavior before hospitalization is independent

$\square$ Tendency to hold back $\square$ Desire to excrete in the toilet

3) Excretion needs

$\square$ Ask for assistance such as "Someone" "Help me" "Nurse"

$\square$ Landing behavior such as side sitting position, standing position, wandering

$\square$ Spontaneous movements to the toilet

$\square$ bowel movements on the bed in inappropriate posture such as the position on the bed

\section{No}

5. Interruption of electrocardiogram and blood pressure monitoring and its risk

1) High risk condition

$\square$ signs of oxygen therapy interruption were seen

2) discomfort and distress

$\square$ Resistance and interruption to electrocardiogram monitoring

$\square$ Sustained blood pressure with automatic measurement

$\square$ Edema of upper limb
1. The patient is in a hypoxic state and at risk of delayed recovery of heart failure.

【The point of nursing aid】

Yes $\square$ Improving pulmonary congestion improves awareness level and comprehension ability. Prevent the patient from worrying about oxygen therapy and prevent interruptions.

1) Reduction of unnatural feeling of NPPV and oxygen mask

2) Reduction of pain and anxiety

3) Devices for reinstallation

2. Cardiac load is not reduced and there is a risk of delayed recovery of heart failure.

【The point of nursing aid】

$\square$ The nurse devises the patient so as not to care about drip

Yes infection and prevents interruption of medication therapy.

1) Communication is taken according to the level of

cognitive function, ensuring peace of mind and safety

2) Distract attention from drip

3) Fixation method by which the patient does not care about

drip infusion

4) Frequent dense observation

5) Risk management

6) Implementation method according to circumstances

7) Response in cases where physical restraint is inevitable

3. When the patient repeats the excretion operation frequently,

cardiac load increases and heart failure may deteriorate.

【The point of nursing aid】

$\square$ If there is a need to continue insertion of the patient's bladder indwelling catheter, the nurse reduces discomfort and prevents self-withdrawal of the patient.

$\square$ After withdrawing the catheter, the nurse devises to satisfy the excretion needs of the patient with the method which minimizes the cardiac load.

1) Determination of necessity of continuation of insertion of bladder indwelling catheter

2) Reduction of discomfort and prevention of uncomfortable feeling of bladder indwelling catheter

3) Strengthening observations to prevent self-extraction

4) Devices for self-removal prevention

5) Assistance that gives a sense of security

6) Risk management

7) Responding to sexual deviant behavior

8) Devices for preventing self-excretion after withdrawal of the bladder indwelling catheter and avoiding excessive cardiac load

4. Movement of the toilet increases cardiac load and heart failure may worsen.

【The point of nursing aid】

$\square$ Satisfy the patient's excretion needs with a method that prevents constipation and minimizes cardiac load

Yes 1) Devices to avoid excessive cardiac load

2) Devices for providing a sense of security

3) Risk management

4) Constipation prevention
5. The monitoring of electrocardiogram and blood pressure is interrupted, and there is a risk of overlooking deterioration of heart failure.

【The point of nursing aid】

Yes $\square$ Devices that patients are not concerned about monitoring electrocardiograms and blood pressure

1) Devices for providing a sense of security

2) Reduction of discomfort

3) Devices that patients do not touch 
6. Interruption of urine volume monitoring and its risk1) High risk condition

$\square$ signs of oxygen therapy interruption

2) Personal background

$\square$ Alone

$\square$ Self-support is strong

3) discomfort and distress

$\square$ There is a self-extraction of the bladder indwelling catheter and its attempt

$$
\text { No }
$$

7. Interruption of oxygenation monitoring and its risk

1) High risk condition

$\square$ signs of oxygen therapy interruption

2) Signs of interruptio

$\square$ Patients worry about wearing

$\square$ The patient is touching the pulse oximeter

$\square$ The patient removes the pulse oximeter

\section{No}

8 . Interruption of rest therapy and its risk for reasons other than excretion needs

1) High risk condition

$\square$ interruption signs of oxygen therapy

2) Personal background

$\square$ Alone $\square$ Self-support is strong $\square$ Tendency to hold back

$\square$ Patients care about family and house

3) Needs

$\square$ Home desire $\square$ Excitement, disturbance, aggression $\square$ Scream

$\square$ Demolition act

4) Physical symptoms and discomfort

$\square$ Sharp increase in weight from before hospitalization

$\square$ Signs of dehydration $\square$ Pain

$\square$ Decrease of swallowing function (risk of fever due to aspiration pneumonia)

$\square$ Environmental discomfort due to temperature, humidity, noise, glare etc.

5) Signs of interruption

$\square$ Landing off behavior, wandering, hyperactivity

$$
\text { No }
$$

9. (Nocturnal) delirium and its risk

1) High risk condition

$\square$ Severity of heart failure

$\square$ The patients take medication for dementia treatment

$\square$ signs of oxygen therapy interruption $\square$ Night of hospitalization day

2) Loss of life rhythm

$\square$ Insomnia of the previous day

$\square$ Day and night reversal (awake in the night and sleep during the day)

$\square$ Night sleep insomnia excluding those who have low sleeping hours and people who sleep slowly

3) Stress against restrictions

$\square$ After examination of cardiac catheter (invasion or restriction of rest)

aIn abstinence

4) Environmental Factors

$\square$ High-intensity lighting at night $\square$ Monitor alarm sound

$\square$ Automatic blood pressure measurement on a regular basis

$\square$ Automatic measurement of periodic pulse oximeter

5) Physical factors

$\square$ Pain due to symptoms of heart failure deterioration

$\square$ Constipation $\square$ Change drugs used

6) Mental factors

$\square$ Anxiety complaint at the time of admission and before the examination

$\square$ Home desire

7) Signs of onset

$\square$ Suspected of delirium in screening for delirium $\square$ Multiple valve

$\square$ restless $\square$ patients cannot keep their rest

$\square$ Decline of motivation and interest $\square$ Slow motion

No

10. Difficulty in catching deterioration symptoms

1) High risk condition

$\square$ Patient wants to be with other people

2) Objective deterioration of heart failure symptoms

$\square$ wheezing $\square$ Lying seat breathing

$\square$ Systolic blood pressure of $130 \mathrm{mmHg}$ or more

$\square$ Decrease in urine volume and urination frequency $\square$ Increase in weight

3) Appearance of BPSD

$\square$ Restlessness $\square$ Excitement $\square$ aggressive

$\square$ Hyperactivity and wandering

$\square$ Destruction behavior when a patient cannot express by words

$\square$ Overdose after intravenous drip reduction and termination

$\square$ Restlessness and excitement after starting meals and ingestion of water
6. Urine volume monitoring is interrupted and there is a risk that the therapeutic effect of heart failure may not be accurately grasped.

【The point of nursing aid】

Incorporate other evaluation methods

1) Assistance giving a sense of security

2) Monitoring using other methods

3) Risk management

7. Oxygenation monitoring may be interrupted and the symptoms of heart failure may not be detected at an early stage.

【The point of nursing aid]

$\square$ The nurse continues monitoring with minimizing patient discomfort.

1) Assistance giving a sense of security

2) Reduction of patient discomfort and innovative devices

3) Devices that patients do not touch

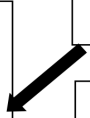

8. Patients cannot keep their rest and suffer from excessive cardiac load and risk heart failure.

【The point of nursing aid】

Yes $\square$ The nurse assesses factors of interruption of resting therapy and copes with the cause.

1) Prevention of interruption of rest therapy

(1) Exploring the reason why patients cannot maintain their rest

(2) relieving anxiety, distress, and discomfort

2) Correspondence when BPSD appears

(1) Assistance to reduce cardiac load caused by overactivity

(2) get cooperation from family members and care providers

(3) Assistance to alleviate anxiety

(4) Drug management

(5) Safety management and risk avoidance

Figure 1. Nursing protocol to prevent discontinuation of treatment in a way other than physical restraint during acute exacerbation of chronic heart failure in patients with impaired cognitive function.

9. Patients are at high risk of developing delirium and risk of delayed recovery of heart failure.

Yes【The point of nursing aid】

$\square$ Nurses provide assistance for prevention of delirium onset and early detection of delirium.

(1) Assistance for early detection of delirium

(2)Assistance for how to spend daytime during hospitalization

(3)Accommodation at night and environment adjustment that

patients can spend with peace of mind

(4) Safety management

(5) Multi-occupational collaboration

(6)Sleeping aid by using drugs with safety consideration

10. The nurse is unable to grasp the symptom of worsening of heart failure of the patient, and the early detection may be delayed.

【The point of nursing aid】

Yes $\square$ Nurses capture the deterioration of heart failure early from the appearance of BPSD and clinical indicators and symptoms. $\square$ Nurses capture the deterioration of heart failure early by utilizing physical assessment and examination data.

(1) Early detection of corruption signs and response

(2) Pain relief at the time of deteriorating heart failure

(3) Prevention against deterioration 
$\square$ To prevent pressure ulcers and skin troubles, use dressings to protect the skin at the site where pressure wounds are likely to develop.

$\square$ Obtain advice from professionals and perform mask fitting to the patient.

$\square$ Attempt to alleviate the discomfort of oxygen therapy; but if it is not alleviated, and if the restlessness continues, ask a doctor about a temporary discontinuation of oxygen therapy. Then, observe the patient's oxygen saturation of the peripheral artery $\left(\mathrm{SpO}_{2}\right)$ value drop, dyspnea, and sweating symptoms and start oxygen therapy.

b) Reduction of pain and anxiety

$\square$ According to the comprehension level of the patient, explain how long oxygen therapy needs to be continued.

$\square$ Assist the patient so that he/she can stay in a comfortable position and alleviate the breathing difficulty.

$\square$ Stay close to the patient as much as possible, touch and show empathic attitude so that he/she can have a sense of security.

$\square$ Provides a long oxygen tube so that it will not disturb the patient's body movement.

c) For reinstallation

$\square$ In order to ensure NPPV, stay with the patient until dyspnea and tachycardia disappear.

$\square \quad$ If the patient removed NPPV, oxygen mask, or canula, do not blame the patient and convince him/her to wear again.

$\square$ Depending on the cognitive function level, explain to the patient repeatedly that NPPV or oxygen mask helps breathing.

$\square$ Explaine that canula is necessary for a cough and put a paper mask so that the patient does not care about oxygen therapy.

$\square$ While the patient is sleeping, gently put NPPV or oxygen mask on the patient.

$\square \quad$ If the patient removes the devices frequently and the hypoxic state continues, let the patient lie down on the side, put the oxygen mask near the mouth, and let it flow.

d) Prevention of aggravation and risk management

$\square$ The patients using adaptive servo ventilation (ASV) or NPPV have severe heart failure; the nurse should frequently visit and observe the patient to prevent severe hypoxia. Alternatively, install a fall preventive movement sensor, so that the nurse can rush to the patient.

$\square \quad$ It is difficult to continue oxygen therapy no matter what. If the patient show agitation, ask the physician and consider the use of a tranquilizer.

$\square$ Oxygen therapy may induce dryness or discomfort of the oral mucosa; prevent upper respiratory infection with oral care and moisturization.

2) Cardiac load is not reduced; there is a risk of delayed recovery of heart failure

[The significant point of nursing aid].

$\square$ Help the patient so that he/she will not be bothered by the IV drip and prevent interruption of medication therapy. 
a) Maintain the sense of safety and security through communications according to the level of cognitive function

$\square$ According to the level of comprehension, explain or visually present what is being done, why the patient needs treatments, and how long it needs to be continued.

$\square$ Explain each time that IV drip will help.

$\square$ Put a bandage around the site of insertion of the IV drip and mark "important". Tell the patient not to remove the line at each visit.

$\square$ Let the patient live freely within the range of at bed-resting.

b) Distract attention from the IV drip

$\square$ Put away the drip bottle, drip line, and drip stand so that the patient will not see them.

$\square$ Let the patient performs activities that they like, which are not too stressful to the body, within the range of bed-resting in order to distract his/her attention.

c) Fixation method by which the patient does not care about IV drip

$\square$ Fix only the site of IV drip so that the patient does not feel distress.

$\square$ Use blood vessels of the upper arm for IV drip, and protect the area with a bandage, net, or soft cine.

$\square$ Put the lines through the neck and fix them not too tightly using a tape.

$\square$ To prevent self-removal, fix the sleeves of the patient's clothes with a tape without touching the skin, so that the patient cannot put his/her hands.

口 To prevent subcutaneous bleeding, do not fix too tightly.

$\square$ Use a wide and difficult-to-remove tape to fix the site so that the connection part of the drip line will not touch the patient's skin.

d) Frequent and dense observation

$\square$ The patient's room should be located near a private room or a nurse station, so that a nurse can have frequent and dense observation.

$\square$ If the patient has difficulty bed-resting, put him/her on a wheelchair so that nurses can observe the patient all the time.

$\square$ Frequently observes the patient and prevent self-removal of the IV drip line.

e) Risk management

$\square$ For a patient treated with drugs which may lower the blood pressure such as Carperitide (Hanp), regularly measure the patient's vital signs and pay attention to sudden decrease of blood pressure. Report to the doctor if the systolic blood pressure drops below $90 \mathrm{mmHg}$.

$\square$ Check if there is no leak with the IV drip. Some patients feel vascular pain when administering antibiotics, so do not use small blood vessels in order to prevent self-removal by the patient.

f) Implementation method according to circumstances

$\square$ If it is difficult to sustain the IV drip, ask the doctor to change it once a day.

- If it is difficult to continue the IV drip even with other methods, ask the doctor if the patient can receive a single dose of diuretic.

$\square$ If the patient has severe edema and if it is difficult to get access to the blood 
vessel from the periphery, ask the doctor if the patient can have a central venous catheterization.

$\square$ Ask the doctor if the patient can receive IV drip in daytime when there are lots of staff around. Physical restraint is the very last option.

g) If physical restraint is inevitable

$\square$ Consider using physical restraint only when the patient has to be alone, such as during nighttime, due to lack of staff members.

口 Consider using physical restraint when self-removal can be dangerous, such as when performing central venous catheterization or IV drips necessary to maintain circulatory dynamics, such as catecholamine from peripheral blood vessels.

$\square$ For physical restraint, select a method which does not disturb the patient's movement.

$\square$ Once physical restraint is performed, discuss when to stop, and release the patient as soon as possible.

3) The patient's cardiac load may increase due to frequent voiding behavior/actions, resulting in aggravation of heart failure

[The significant point of nursing aid]

$\square$ If there is a need to continue insertion of the bladder indwelling catheter, reduce discomfort and prevents self-removal by the patient.

$\square$ After removing the catheter, attempt to satisfy the patient's need on urination with a method minimizing the cardiac load.

a) Judge if the patient needs insertion/continuation of the bladder indwelling catheter

$\square$ In patients with decreased cardiac function (eg, LVEF $20 \%$ or less, with an underlying disease associated with aortic stenosis), careful judgment and approach is required as he/she may develop loss of consciousness with cardiac load.

$\square$ Use a bladder indwelling catheter for patients receiving diuretics such as Hanp or Furosemide (Lasix) via IV drip. Consider removing the catheter if the prescription is changed to oral drugs.

$\square \quad$ If the level of the cardiac function is less than 3 METs (ambulatory), continue the bed-resting therapy without removing the bladder indwelling catheter as much as possible for early recovery.

$\square$ For the patient with cardiac function level of 3 or above with METs, ask the doctor if the patient needs the catheter.

$\square$ The catheter can be removed if the patient can use a portable toilet on the bed side or if he/she can do stepping exercise for more than 20 times in the sitting position at rehabilitation.

$\square$ Even if the patient feels restless, leave the catheter in and observe as long as it is easier this way for the patient to void.

b) Reduction and prevention of discomfort and uncomfortable feeling for bladder indwelling catheter 
$\square$ Choose 14 Fr or less for the size of the bladder indwelling catheter.

$\square$ Use the bladder indwelling catheter made of silicone with a large amount of lubricant.

$\square$ Do not leave the patient's pain or discomfort untreated. Use anti-inflammatory analgesics or sedatives to resolve the problem.

$\square$ For male patients, fix the catheter to the lower abdomen, where it is not likely to disturb the body movement, and avoid friction in the urethra to prevent development of ureterocutaneous fistula.

$\square$ For female patients, fix the catheter at two sites, the thigh and lower abdomen, to reduce friction and discomfort.

c) Reinforce observation to prevent self-removal

$\square$ Pay attention to excretion behavior after intravenous injection of diuretics especially within the first 30 minutes.

$\square$ In patients with bladder indwelling catheters, there is always a risk of self-removal; Carefully observe the timing when a large amount of diluted urine flows out after intravenous injection of a diuretic.

d) For self-removal prevention

$\square$ Depending on the level of the cognitive function, write down on a piece of paper "Please press a nurse call button if you need to go to the toilet", and post it at the place where the patients can see, and tell the patients directly if necessary, or use the fall preventive movement sensor.

$\square$ When the diluted urine flows out, strongly rub the indwelling catheter with hands to show that the urine outflow is sufficient.

$\square$ Let the patient wear pajama pants, make sure that the patient's hands do not touch the bladder indwelling catheter and pull out the lines from the lower side of the pants around the feet.

$\square \quad$ If the patient keeps going to the toilet even with bladder indwelling catheter, consider moving the patient to another bed near the toilet.

$\square$ For patients sticking to excretion, talk about topics other than excretion and divert attention.

e) Assistance that gives a sense of security

$\square$ According to the level of comprehension, explain to the patient how long he/she will need the bed-resting therapy and the bladder indwelling catheter.

$\square$ The patient may have unpleasant memory of the indwelling catheter. Explains to the patient that it is for the temporary use.

$\square$ Approach from the front, talk to the patient with calm and gentle voice without making him/her feel panic.

$\square$ The patient who needs to go to the toilet frequently may have anxiety. So stay with the patient at least once in three times, talk to him/her and find the cause, so that the patient can have a sense of security.

$\square$ Be aware that patients who frequently go to the toilet but cannot not void may feel troublesome for others.

f) Risk management

$\square$ Reduce the amount of fixed water in the bladder indwelling catheter to about 
2 to $3 \mathrm{cc}$ (to prevent urethral damage during self-removal).

$\square \quad$ If the patient urinates frequently but in a small quantity, check for signs of urinary tract infection.

$\square$ In the patient with severe heart failure, the condition can be exacerbated by urinary tract infection; Try to prevent infection by removing the bladder indwelling catheter in an early stage.

$\square \quad$ If a male patient complains of urinary frequency after removal of the urethra indwelling catheter but the urination volume is small, suspect prostate hyperplasia and speak with the doctor.

g) For sexual deviation behavior

$\square$ If the patient has paranoia for excretion or shows sexual deviation behavior, a male nurse should be in charge of this patient.

$\square \quad$ If sexual deviant behavior is induced by irritation of the bladder indwelling catheter and the patient cannot maintain rest, consider removing the catheter.

h) For preventing self-excretion after removal of the bladder indwelling catheter and avoiding excessive cardiac load

$\square$ If it is difficult to continue using the bladder indwelling catheter to a patient, consider removal and assist in the following order: 1) Use diapers on excretion on the bed, 2) Use urine bottle or toilet bowl for excretion on the bed (use urinal comfort for a male patient), 3) Use portable toilet at the side of the bed, 4) toilet on the wheelchair.

$\square$ Explain to the patient that urinary device is an excretion tool to be used on the bed while resting.

$\square \quad$ If the patient cannot void in the standing position or outside the toilet, assist urination using urine bottle, portable toilet, or toilet for wheelchair for the first few times while observing signs for tachycardia or hypoxia.

$\square$ Monitor the time for behaviors and actions associated with the voiding needs, finds a sign of voiding, and assist urination.

$\square \quad$ If a patient wearing a diaper removes the tube, ask for the reason and try to satisfy his/her needs.

$\square$ Write down on a piece of paper at what time the patient voided last time, and let the patient know that he/she just finished voiding by showing a clock and the paper.

$\square \quad$ If the patient frequently shows his/her desire to void, try to measure the urine volume in the bladder using a bladder scan and consider if urination induction is necessary.

4) Moving to the toilet may increase cardiac load and aggravate heart failure.

[The significant point of nursing aid]

$\square$ Satisfy the patient's voiding needs with a method that prevents constipation and minimizes cardiac load

a) For avoiding excessive cardiac load

$\square$ Ask the excretion status of the patient before hospitalization to the caregivers, and take actions if constipation continues. 
$\square$ If the excretion status before hospitalization is unknown, performs a physical assessment of the abdomen and take actions if constipation is suspected.

$\square$ Explains about necessity of excretion on the bed to the patient, and attempt to assist excretion using a toilet bowl or a diaper.

$\square$ If the patient is resistant to diapers or toilet bowls, consider other methods such as siting on a toilet placed on the bed.

$\square \quad$ If it is difficult for the patient to excrete in the supine position with respiratory distress or if the patient is does not feel satisfied with excretion on the bed, let the patient use the portable toilet with full assistance.

$\square \quad$ If the patient refuses to use the portable toilet, takes the patient to the toilet with full assistance.

$\square \quad$ In order to ensure patient safety, stay aside until the patient finish voiding while protecting his/her privacy.

$\square$ Depending on the level of the cognitive function, write down on a piece of paper "Please press a nurse call button if you need to go to the toilet", and post it at the place where the patients can see, and tell the patients directly if necessary.

$\square$ Explain about stool extraction and perform the procedure with consent. If the patient has been extracting stool by him/herself, take over the procedure with permission.

b) For providing a sense of security

$\square$ Depending on the level of the cognitive function, explain for how long the patient will need to excrete on the bed.

$\square$ After excretion, let the patient to see the excrement.

c) Risk management

$\square$ When a patient sticks to defecation, divert the patient's attention to other things such as radio or music which is not stressful to the patient's body, while monitoring the patient. The cardiac load can increase by up to 20 in terms of the heart rate and blood pressure.

$\square$ Even if the patient sticks to defecation and asks for an enema, the patient in the acute phase may develop shock, so give the patient suppository medicine or perform stool extraction.

$\square$ Patients with decreased cardiac function (e.g., LVEF $20 \%$ or less, with an underlying disease associated with aortic stenosis ) have a risk of causing loss of consciousness when cardiac load is applied, so the nurse carefully correspond.

d) Constipation prevention

$\square$ Prevent constipation of the patient (by using laxatives, abdominal massage, warm pad, leaving the bed soon, etc.).

5) Electrocardiogram and blood pressure monitoring is interrupted; there is a risk of overlooking deterioration of heart failure

[The significant point of nursing aid].

$\square$ For making patients not too concerned about monitoring electrocardiograms and blood pressure 
a) For providing a sense of security

$\square$ According to the level of comprehension of the patient, explain how long the patient will need to wear the electrocardiogram monitor.

$\square$ As the patient's life history may be associated with the situation, provide the patient with alternative ways.

(e.g. A former fisherman may show a particular behavior such as pulling the lines connected to the monitor as if these are the fishing net).

b) Reduction of discomfort

$\square$ Apply stickers of the ECG monitor to the back of the patient.

$\square \quad$ Use non-irritative stickers.

$\square$ The patient may feel itchy; keep the skin clean and moist and use non-irritative electrodes/stickers.

$\square$ Put the lines or cords away so these do not touch the patient's skin.

$\square \quad$ Put the lines and cords through the abdominal side.

$\square \quad$ Keep the monitoring sound low.

$\square$ If edema is observed in the upper extremity, measures blood pressure manually each time instead of using an automatic blood pressure measuring device.

c) For making the patients not to touch

$\square$ Fix the patient's shirt around neck using a tape so the patient cannot put of his/her hand inside.

6) Urine volume monitoring is interrupted; the therapeutic effect for heart failure may not be accurately measured

[The significant point of nursing aid].

$\square$ Use other evaluation methods

a) Assistance giving a sense of security

$\square$ According to the level of comprehension of the patient, explain how long the patient needs to use the bladder indwelling catheter.

b) Monitoring using other methods

$\square$ If urine volume measurement is not reliable, assess congestion or edema next morning by measuring the patient's weight.

$\square$ Ask the physician and assess weight, edema, moisture payment, dyspnea, malaise, and inspection results.

$\square$ If the patient needs to stay in the bed, use the weight measuring device on the bed.

$\square$ Depending on the level of rest, measure the patient's weight on the bed side.

$\square$ For the ambulatory patient, discuss with the physician if he/she needs to accumulate urine using a device. Use a toilet with a function of measuring urine volume, if necessary.

c) Risk management

$\square \quad$ The nurse must observe the patient once every 15 to 30 minutes after starting the IV drip, and then every 30 minutes thereafter.

$\square$ There is a risk of damaging the urethra of the patient removes the bladder indwelling catheter by him/herself. Hence, do not reinsert the catheter and 
use other excretion styles such as using diapers. Monitor the patient using index other than urine volume measurement.

7) Oxygenation monitoring may be interrupted; symptoms of heart failure may not be detected at in the early stage

[The significant point of nursing aid].

$\square$ Continue monitoring while minimizing patient discomfort.

a) Assistance giving a sense of security

$\square$ Depending on the level of comprehension of the patient, explain to the patient how long they have to perform the oxygenation monitoring.

b) Reduction of patient discomfort and concerns

$\square$ Long-term oxygenation monitoring may cause discomfort and skin integrity disorder in patients. Unless the patient has received tracheostomy or ventilator, ask the patient if they can do monitoring only when needed rather than constantly and explain the reason.

$\square$ Changes the monitoring or measuring sites accordingly.

$\square$ If the patient refuses the measurement, use oxygenation index other than $\mathrm{SpO}_{2}$.

c) For making patients not to touch

$\square$ For patients who cannot walk, attaches the device on their toes.

8) Patients cannot stay rested and suffer from excessive cardiac load; a risk of aggravation of heart failure

[The significant point of nursing aid].

$\square$ Assess factors for interruption of bed-resting therapy and deal with the cause.

a) Prevention for interruption of bed-resting therapy

1) Explore the reason why the patient cannot stay rested.

$\square$ Check the past medical history as well as the history of drug side effects.

$\square$ Observe the patient's distress including physical pain, constipation, insomnia, and hunger.

$\square$ Observe mental distress associated with sadness, anger, loneliness, etc. as well as personality of the patient him/herself.

$\square$ Observe environmental distress associated with sound, light, taste, smell, the air conditioning, humidity, and sensation.

$\square$ Observe distress associated with relationships with others including families/caregivers and nurses, etc.

$\square$ Observe the gap between the patient's life history or the previous life style and current situation.

2) Relieving anxiety, distress and discomfort

$\square$ Depending on the level of comprehension, explain for how long the patient needs to continue the bed-resting therapy.

$\square \quad$ Let the patient stay in a comfortable posture and fix their clothes.

$\square \quad$ If there is pain in the patient, the nurse actively controls pain.

$\square \quad$ If the patient is suffering from pain, perform an active control of the pain.

$\square$ If the patient is sweating due to difficulty breathing, apply a soft cold pat around the patient's neck and adjust the coverture. 
$\square$ Provide a comfortable environment by adjusting temperature, humidity, sound, and light, etc.

b) If BPSD appears

1) Assistance to reduce cardiac load caused by overactivity

$\square$ If there is a bathroom or toilet nearby, the patient who used to be independent may feel like using it. So the patient should be move to a place where he/she cannot see the bathroom or toilet.

$\square$ Listen to the patient's complaints about hyperactivity, wander and anger, etc. and do not force them to rest. Attempt to satisfy the patient's needs while paying attention to the cardiac load by observing or staying with the patient on a wheel chair while he/she is wandering around.

$\square$ Whenever a nurse moves the patient on a wheelchair, continue oxygenation therapy to prevent deterioration of heart failure. Keep assisting the patient according to his/her needs but pay attention to the patient's conditions so that he/she will not become overactive. Takes the patient around the ward and suggest to go back to the room once he/she calmed down.

$\square$ If a wanderer refuses to get on a wheelchair, leave the chair in the hallway so that the patient can take a rest at any time. Ask the patient to rest when passing bay in front of the patient, or say "let's take a break, you must be tired" after wandering.

$\square \quad$ If the patient is angry, encourages independence within the allowable resting level and do not give excessive assistance. (e.g. Ask the patient to clean the table after eating, or ask the patient to blush their teeth etc.)

$\square \quad$ If the patient is excited, continue observation without any intervention.

$\square$ In patients with decreased cardiac function (LVEF $20 \%$ or less, with an underlying disease associated with aortic stenosis), there is a risk of causing loss of consciousness when cardiac load is applied, and nurses respond carefully.

2) Obtain help from family members and caregivers

$\square$ Ask family members and caregivers to see the patient and take a walk with the patient on a wheel chair.

$\square$ In front of the patient, ask the family members and the caregivers not to show that they are feeling troubled because of the patient.

$\square$ Ask the family members and the care givers to call the patient even they cannot visit, so that the patient will feel secure.

$\square \quad$ Tell the family members and the care givers that BPSD is a temporary symptom and that the patient is feeling grateful for their support.

3) Assistance to alleviate anxiety

$\square$ Ask the patient to share stories of his/her life and listen to them until he/she calm down.

$\square \quad$ If patient feels restless, stay close, show sympathy and touch him/her gently.

$\square$ If the patient has excitement, restlessness, wandering, or overactivity, the nurse is accompanied and involved in one-on-one. Still, if those symptoms are not alleviated, different nurses deal with it.

$\square$ If the patient feels restless to stay alone in the hospital room, let the patient 
read newspapers or magazines at the nurse station.

$\square \quad$ If the patient wanders at nighttime, stay with the patient and talk until he/she calms down and falls asleep.

$\square$ If distress associated with deterioration of heart failure is not the reason, divert the patient's attention by changing topics.

$\square$ Create a space where the patient can put something he/she likes.

4) Drug management

$\square$ Observe the clinical examination results and perform the physical assessments to see if the patient feels restress or agitated due to physical factors such as electrolyte abnormality and dehydration. Do not prioritize use of drugs when the patient is feeling restless or agitated.

$\square$ Suspect BPSD or delirium when the patient is restless or agitated and consider using drugs when he/she no longer accepts explanation. listens to the nurse. Carefully examine the past medical history and drug side effects, and find the most appropriate treatments.

$\square$ Assesses the cause and consider the use of appropriate medication while paying attention to side effects.

(e.g. For causes of BPSD and delirium, appropriate drugs should be used for anxiety, circadian rhythm disorder, insomnia due to symptoms associated with aggravation of heart failure).

$\square$ The doctor's prescription for restlessness or excitement may not be appropriate for heart failure; ask the doctor what the best treatment would be for the patient.

$\square$ Once the patient becomes angry, the cardiac load is applied and oral administration will be difficult; use the antipsychotic drugs at appropriate timing.

$\square \quad$ If sleeping pills are used at nighttime after BPSD or delirium appears, the patient's circadian rhythm may be disturbed. Therefore, consider prophylactic administration of drugs at appropriate timing such as during daytime or at dinner time.

$\square$ Be careful not to reduce the level of the patient's activities of daily living (ADL) by continuously using the drugs when there is no symptom.

$\square$ Patients with Lewy body dementia are sensitive to antipsychotics and sleeping pills, so pay special attention when using these drugs.

5) Safety management and risk avoidance

$\square$ Let the patient stay in a private room or in a room close to the nurse station. Observe the patient carefully with a fall preventive movement sensor.

$\square$ Makes the bed arrangement similar to the position that he/she used to have before hospitalization so that the patient will be able to get on and off the bed from the same side.

$\square$ Have the patient use a low bed or wear HIP PROTECTOR to prevent fractures due to falls.

$\square$ Do not physically restrain the patient from leaving the bed behaviors. Place security guards around the bed from 3 sides so that the patient can get on and off the bed on just one side. Use a fall preventive movement sensor and 
safety mat to prevent fall event.

$\square$ Considers use of tranquilizer until the patient's condition is settled. Use a safety belt or safety band on the patient, if necessary.

$\square$ Know which drugs can cause falls.

$\square$ BPSD may be induced by aggravation of heart failure due to aspiration (infection, fever) or dehydration in patients with decreased swallowing function; Perform oral care and prevention of dehydration.

6) Patients are at high risk of developing delirium; a risk of delayed recovery of heart failure.

[The significant point of nursing aid]

$\square$ Nurses provide assistance for prevention and early detection of delirium.

a) Assistance for early detection of delirium

$\square$ If the patient shows lack of motivation/interest and/or loss of appetite, perform a physical assessment to examine hypo-active type delirium and deterioration of heart failure symptoms.

b) Assistance for adjusting the life-style rhythm

$\square \quad$ Let the patient know the today's date.

$\square$ Adjust the circadian rhythm and prepare the patient for appropriate sleep at nighttime by moving the bed near the window or under the sun or by using curtains to stimulate the circadian rhythm.

- Perform light therapy and adjust the patient's biological rhythm.

$\square$ Put the patient on a wheelchair and take a walk to change the patient's mood. If the patient can stay in the sitting position, maintain his/her activity level within the resting state such as by eating meals on the wheel chair.

$\square$ Expand the patient's activities during the day according to the progress of the patient's rehabilitation.

$\square$ Creates a life rhythm and prepare the patient for sleep at night time by incorporating hobbies, activities, and recreation which last for a short period of time (within 30 minutes) while monitoring heart rate, blood pressure, and $\mathrm{SpO}_{2}$. Activities should be avoided however before and after other activities such as eating meals, excretion, and taking care of appearance.

(1) Bed-resting and fasting period: activities of about 1.0 METS such as watching TV, listening to music and radio.

* The cardiac load can increase by up to 20 in terms of the heart rate and blood pressure.

(2) Meal-intake and washing face possible in the sitting position: activities of 1.3 - 1.5 METS such as reading a book or newspaper, knitting, doing handicrafts, calligraphy, and painting etc.

c) Preparation of environment where patients can stay peaceful during day and night

$\square$ Stay with the patient until he/she falls asleep, while preparing reports in necessary.

$\square$ Patients may feel anxious especially at night. Prepare environment where the patient can sleep well such as by avoiding light, footsteps, sound of pulling a 
cart, room temperature, bedding, taking vital signs when necessary without using an automatic device, etc.

$\square$ Even for the patient who had been independent before hospitalization in terms of $\mathrm{ADL}$, ask families and caregivers to stay with the patient until the bedtime, if possible.

d) Safety management

$\square$ If there is a risk of falling, use a fall preventive movement sensor and a safety mat.

$\square$ If the patient becomes too aggressive with his/her personal belongings around, take those away out of the reach.

e) Multi-disciplinary collaboration

$\square$ Ask the doctor to arrange for consultation with the liaison team in the psychiatry unit.

$\square$ When using drugs for insomnia, record the patient's condition and side effects in details so that they can evaluate the effects.

$\square \quad$ If there is no prescription for delirium or BPSD which may develop during night time, ask the doctor for direction.

$\square$ Tell the families and caregivers that delirium is a temporary symptom, so that there is no problem going home after discharge.

f) Sleeping aid using drugs with safety consideration

$\square$ First of all, assesses if the patient cannot sleep even though he/she wants to sleep or because of anxiety. Consider giving sleeping pills in the former case, and anti-anxiety drug in the latter.

$\square$ Know the sleep pattern from the patient's life history and consider if he/she needs the sleeping pills. (e.g. There is no need of take pills if the patient used to go to the bed at $10 \mathrm{pm}$ and wake up at $4-5 \mathrm{am}$.)

$\square \quad$ The use of sleeping pills after the onset of delirium may not be effective; consider using the drugs as soon the signs were observed.

$\square$ In case of agitated, aggressive and violent patient, priority is given to drug administration over nursing care. Liquid medicine can be useful for oral intake as it can be absorbed, in a small amount, from the mucosa even if the patient refused to take.

$\square \quad$ Use sleeping medication only at night time so that its effects will not be seen in daytime. (e.g. Stop IV drip as soon as the patient falls asleep.)

$\square$ Basically, do not use sleeping pills after midnight or at 4 o'clock in the morning just because the patient woke up.

$\square$ Selects appropriate drugs from the prescription and use them carefully.

$\square$ The patient with Lewy body dementia tends to have adverse reactions. Carefully judge if the patient must be treated with the drug.

10) The signs of aggravation of heart failure are overlooked; delayed detection. [The significant point of nursing aid]

$\square$ Detect aggravation of heart failure in the early stage from the onset of BPSD, clinical index and symptoms and take necessary measures. 
$\square$ Detect aggravation of heart failure in the early stage by utilizing physical assessment and examination data.

a) Early detection and actions taken against signs of aggravation

$\square$ Find out the area and the degree/type of severity of the symptoms (e.g. the patient is suffering from shortness of breath).

$\square$ Patients with mild dementia may have subjective symptoms, so obtain subjective information as well.

$\square$ Nurses who know the patient's characteristics well, such as primary nurses, should be in charge.

$\square$ Observe unusual symptoms such as cold sweats, restlessness, wandering, etc.

$\square \quad$ If aggressive and overactive BPSD appears suddenly, suspects acute exacerbation of chronic heart failure and assess signs of aggravation.

$\square$ In addition to complaints of patients, obtain data through physical assessments, clinical examinations, as well as indicator of aggravation of heart failure such as edema and change in appetite and activity, etc. Conduct assessments on these factors and detect the aggravation signs of heart failure in the early stage.

$\square$ In patients using diuretics, detect the signs of dehydration and renal dysfunction in the early stage.

$\square$ In patients complicated by hypertension, cardiac load may increase along with rapid increase of blood pressure, resulting in aggravation of pulmonary edema. Continuously perform prevention for coldness and constipation as well as blood pressure monitoring.

$\square$ In patients complicated with anemia, the amount of myocardial oxygen consumption increases due to elevated heart rate and cardiac output; continuously monitor subjective symptoms and objective factors.

b) Alleviating distress during aggravation of heart failure

$\square$ Assist the patient to find a comfortable position.

$\square$ If the patient has difficulty breathing or physical distress, ask the doctor if the patient should take sedatives, etc.

$\square \quad$ If the patient has difficulty in verbal communication and changing the position from sitting to lying on the back, he/she may be under the hypoxic state. Consider that the patient is suffering from respiratory distress and observe respiratory condition, cyanoses, and $\mathrm{SpO}_{2}$ to take necessary measures.

$\square$ Improve peripheral circulation with foot/hand bath.

c) Prevention against deterioration

$\square$ The patient tends to become overactive after reduction or completion of IV drip. Explain to the patient that they should do activities within the range of resting.

$\square$ Ask the patient to eat slowly at a small quantity without putting too much in the mouth when they eat meals so that they will not have cardiac load.

$\square$ Do not let the patient do any physical activities following meals, excretion, or hygienic care. 


\section{Discussion}

\subsection{The Process of Nursing Protocol Preparation}

Based on the evidence from the previous study (original draft) and opinions of certified nurses specialized in chronic heart failure and/or dementia care, we prepared a revised draft of the nursing protocol using a mail survey on nurses working in clinical settings for the effects of assessment items and specific nursing care practice. The nursing protocol was considered useful by $93.9 \%$ of these nurses, suggesting that it is valid to a certain level.

\subsection{Usefulness of the Nursing Protocol}

\subsubsection{For Improving Quality of Nursing Care}

The nursing protocols created in the previous studies include the protocol for the elderly demented patients with wandering behavior [6], the nursing protocol for safe and efficient increase of the resting level in acute stroke patients [7], and the protocol for safe and easy step-wise change in the posture angle from $30 \mathrm{de}-$ grees to the open back position in acute stroke patients [9]. For dementia patients complicated by physical disorders, BPSD and delirium are likely to develop in the acute exacerbation phase of the physical disorders. However, no one has ever attempted to create a nursing protocol aiming for conducting clinical examinations and treatments smoothly in patients with impaired cognitive function and physical diseases so that they can have a speedy recovery. The nursing protocol created in this study is something that they had been waiting for in the clinical setting and therefore meaningful. Moreover, this is created to provide nursing care for patient in acute phase of chronic heart failure using methods other than physical restraint. Actually, in the second stage of this study, there was also an opinion that the certified nurses "we want to use the nursing protocol as soon as possible. It will be helpful." We believe that by using this protocol in the clinical setting, the quality of nursing care for demented patients with physical diseases will improve.

\subsubsection{Usefulness as a Risk Management for Medical Accidents}

We considered that this type of protocol will contribute to standardization of nursing care, which they have been waiting for BPSD such as wander and hyperactivity are likely to develop when the patients' physical conditions are aggravated [9]. Hence, we should regard BPSD as a sign of aggravation of the patients' heart failure. There was also a comment that we should include the basic contents for newly graduated nurses. We consider that this protocol will be useful to acquire knowledge as well. If we cannot predict a risk for patients with impaired cognitive function in the acute exacerbation phase of chronic heart failure, they may wander and have fall accidents, etc. Then, we may end up being in troubles associated with management responsibility [10]. The number of accidents associated with self-removal of blood access or falling from the bed has been also increasing [11]. Hence, it is important to manage the risk of patients with impaired cognitive function, receiving medical treatments. By implement- 
ing this nursing protocol, we believe that it will be possible to provide a certain level of preventive care to the patients, and that it will be also be useful as a risk management tool to prevent medical accidents.

\section{Conclusion}

It was confirmed that this nursing protocol was useful for preventing discontinuation of treatment by methods other than physical restraint during acute exacerbation of chronic heart failure in patients with impaired cognitive function. In addition, it helped to standardize nursing care in order to predict potential risks for patients with impaired cognitive function. In order to generalize this research result, it is necessary to increase the number of target nurses and further verify the usefulness of nursing protocols.

\section{Acknowledgements}

We would like to express our sincere gratitude to all the nurses who participated in this study, especially those specialized in chronic heart failure and/or dementia care for helping us with interviews, as well as those from all over the country for participating in the questionnaire survey.

\section{Funding}

This research was conducted with the aid of 2015-2018 Scientific Research Grant Subsidized Project Basic Research (C) (Issue No. 15 K11577).

\section{References}

[1] Otsu, H., Moriyama, M. and Makaya, M. (2013) The Realities Regarding Difficult Nursing Support for Elderly Demented Patients with Deteriorating Chronic Heart Failure. The Journal of Japanese Association of Cardiovascular Nursing, 8, 26-34.

[2] Ministry of Health, Labor and Welfare (2017) Medical Reimbursement for 2016. 206-208.

http://www.mhlw.go.jp/file/05-Shingikai-12404000-Hokenkyoku-Iryouka/00001123 $\underline{06 . p d f}$

[3] Otsu, H. (2015) Difficulty and Effective Nursing Care of Demented People with Chronic Heart Failure for Certified Nurse in Dementia Nursing. The Journal of Japanese Association of Cardiovascular Nursing, 10, 64-74.

[4] Otsu, H., Inoguchi, T., Moriyama, M., Takayama, S., Watanabe, Y. and Kume, M. (2018) Characteristics of Patients with Decreased Cognitive Function Undergoing Treatment for Acute Exacerbation of Chronic Heart Failure-Basic Survey for Standardization of Nursing to Prevent Discontinuation of Treatment. Health, 10, 667-690. https://doi.org/10.4236/health.2018.105052

[5] Guideline on Diagnosis and Treatment of Cardiovascular Disease (Report of Joint Research Group of 2010): Guidelines for Treatment of Acute Heart Failure (JCS 2011). http://www.j-circ.or.jp/guideline/pdf/JCS2011_izumi_h.pdf

[6] Otsu, H., Takayama, S. and Watanabe, Y. (2013) An Examination of the Utility of Protocol in Dealing with Wandering for Elderly People with Dementia. Journal of Health Science Research, 3, 85-99.

http://hoken-kagaku.com/journal/Vol3(2013).pdf 
[7] Nakazawa, A. (2013) An Attempt to Introduce a Protocol to Increase the Resting Degree by Nurses Led to Acute Stroke. Brain Nursing, 29, 53-57.

[8] Kobayashi, Y., Yano, S. and Koga, M. (2015) The Creation and Investigation of the Efficacy of Safe and Comfortable Dorsal Open Sitting Position Protocol for Patients with Acute Cerebral Hemorrhage Patients. Journal of Japanese Academy of Neuroscience Nursing, 3, 23-31.

[9] Otsu, H., Tamada, S., Kudo, M. and Ogasawara, E. (2016) Fundamental Inquiry to Consider a Way of Nursing Care for Demented Elderly with Physical Disease. Journal of Health Science Research, 6, 13-28. http://hoken-kagaku.com/journal/Vol6(2016).pdf

[10] Nagano, N. (2013) Medical Care and Litigation Risk of Elderly with Insufficient Cognitive Function. Japanese Journal of Geriatric Psychiatry, 24, 568-575.

[11] Kurihara, S. (2016) Current Situation and Medical Accident of Elderly Dialysis Patients. Journal of the Saitama Society for Dialysis Therapy, 5, 58-60. 